\title{
Research on Computer Network Teaching Based on Artificial Intelligence
}

\author{
Zuqin $\mathrm{Mo}^{1, \text { a }}$ \\ ${ }^{1}$ Department of Information and Intelligent Engineering of Hubei Industrial Polytechnic, Shiyan, \\ Hubei, China, 442000 \\ aemail,
}

Keywords: Computer Network Teaching, Artificial Intelligence, Research

\begin{abstract}
With the development of technology change and the establishment of people's concept of lifelong learning education system, especially the popularity of computer networks, computer network education as a new educational model is entering a new stage of rapid development. However, a lot of confusion and difficulties encountered in practice to people's learning is a big obstacle. Application of artificial intelligence computer network education, to give people hope. This article describes the theory and techniques of artificial intelligence, and its specific application in the computer network teaching.
\end{abstract}

\section{Introduction}

AI (Artificial Intelligence) is to use artificial methods in a machine (computer) to implement intelligence or human intelligence on the machine so that the machine analog or people have human-like intelligence. It is developed on the basis of neurophysiology, philosophy of language, control theory, information theory, computer science and other disciplines on a subject. Now artificial intelligence research is often used in three ways: First, the use of biological methods to ascertain the nature of human intelligence; second is to use computer science research method, which uses a network connection to connection-oriented mechanism, the use of artificial neural networks, simulate human thinking in images, the method can solve the difficult knowledge of notation, the third is the use of physiological methods. From the perspective of computer application systems, artificial intelligence is the study of how to make a smart or intelligent machine system, the ability to simulate human intelligence activities, to extend people smart science.

\section{The Status of the Computer Assisted Instruction}

Computer technology in teaching known as computer-assisted instruction, CIA with respect to the traditional teaching is a major change on teaching methods, but with the continuous development of teaching, the traditional computer multimedia teaching mode gradually lagged behind the requirements of the times. Its deficiency is mainly reflected in the following aspects.

Poor Interactivity Ability. Comparison of existing computer-aided teaching mode monotony, in the actual teaching activities, primarily as computer applications or new materials science and technology board only, most teachers will use the already engraved disc, the teaching materials through a computer screen is displayed, the process is rigid curriculum, the role of the computer at this time it is only an electronic blackboard. Therefore, in the actual classroom, the teacher is actually only a predetermined process operation, student lectures patterns still stuck in the traditional lecture mode.

Lack of Intelligence. In teaching, because the children are learning and mastery of knowledge vary, student learning initiative also vary, requiring computer assisted instruction intelligence to automatically provide information to students, giving them a selective learning. Teachers teaching only actively participate in the study to achieve better teaching results can be provided through intelligent computer services\% individualized to the greatest extent possible and promote teaching. Teaching effectiveness based, very necessary to improve the intelligence of multimedia teaching.

Lack of Extensive Features. This is the first computer-assisted instruction inherent flaw in the 
design of the beginning of it is the overall design of a particular field of knowledge-based answers to questions by\% of teaching content and design, to render the original design of the system allows the contents of knowledge within the scope of this It cannot be based on the actual situation of the students and teachers to arrange for students to different teaching contents, unable to guide students according to students' cognitive characteristics and optimal learning.

Lack of Openness. Openness is the lack of multimedia teaching serious problem. Fixed teaching content to adapt to the narrower, rigid classroom plans and arrangements, the lack of independent initiative, since the fixed teaching resources cannot update features make the teaching content cannot change, cannot choose the content for students features.

\section{Research on Artificial Intelligence}

The so-called artificial intelligence is the use of artificial intelligence methods to achieve on your computer, it can be said is a simulation on the computer AI. At present, the presence of three artificial intelligence research ways: First, to support the theory of biology, human intelligence to grasp the essence of the law; the second is to support computer science, intelligent simulation through artificial neural networks, human-machine interaction; third is biological theory as support. Smart technology is mainly divided into two categories, human and computer intelligence, there is a complementary relationship between the two. The use of artificial intelligence techniques to achieve human intelligence into machine intelligence to the contrary, it is possible to use machine intelligence into human intelligence intelligent tutoring.

The AI has very strong search capabilities. This function is related to the use of searches search technology for rapid retrieval and information to meet individual information needs; secondly, artificial intelligence, knowledge representation with a strong ability. Specifically, the artificial intelligence of the behavior information, it is possible the same as human intelligence, fuzzy information to be represented; and finally, with strong artificial intelligence and speech recognition capabilities abstract. The former is mainly to be dealt with fuzzy information. While the latter mainly to distinguish between the degrees of importance of the information, in order to improve the efficiency of information processing. Users only need to make intelligent machines can be specific requirements, as complex solutions on to the smart program.

The man-machine dialogue is more flexible. Traditional multimedia human-computer dialogue largely inadequate, resulting in tedious teaching, we cannot achieve the desired good results, but the smart media is not the case, he was able to achieve human-computer interaction and free dialogue, while combining the students 'practical to give different students' questions level answer. Secondly, teaching feasibility stronger. As students in terms of cognitive ability and personal qualities there are differences, and motivation to learn are not the same, AI must be combined with students' actual learning situation, to develop individualized learning plans and learning objectives for every student design, students highly targeted teaching, truly individualized. The former belongs to the salient features of artificial intelligence, and important aspects of the performance of the latter part of artificial intelligence. Finally, intelligent multimedia features with a teacher. In the actual teaching process, intelligent multimedia teaching can intelligently evaluate the conduct of the parties to be able to detect weak points in teaching, contributing to teaching and learning, improve teaching quality and effectiveness.

\section{The Application of Artificial Intelligence Technology in Computer Network Education}

Intelligent Decision Support System (IDSS). IDSS in the early 1980s suggested that DSS is a product of the combination with the AI. Basic member IDSS system for the database, model base, method base, man-machine interfaces and smart components can be composed of members from these basic structures and four fusion structure of the two systems architecture. Currently, intelligent decision support system IDSS has become the development direction of the decision support system DSS, the application of the network in the field of education showing strong growth potential and a bright future. For example IDSS widely used in the digital library, the system is able to provide the 
necessary decision-making data, information and background material for policy-makers to help clear decision-making goals and identify issues, create or modify the decision model, offers a variety of alternatives programs, and programs to evaluate and optimal selection, through interactive features to analyze, compare and judge, to provide the necessary support [7] for the correct and effective decision making.

Intelligent Teaching Expert System (ITES). ITES provides an intelligent teaching environment, which uses computers to simulate the process of teaching thinking experts and professors, using a variety of teaching methods advanced AI technologies and multimedia interactive teaching system formed open, students can take the initiative to obtain knowledge of the system, the system It can be individualized instruction based on the student's knowledge, abilities and learning methods in order to achieve the best teaching effect, the real unmanned aim individualized adaptive teaching. ITES to ICAI based. It is based on cognitive science and scientific thinking as the theoretical basis, a comprehensive knowledge of artificial intelligence technology, education, psychology and other subjects for students to implement a new educational technology and effective education. ICAI's role is to learn by studying the characteristics of human thinking and processes, seeking to learn the cognitive model, so that students get individualized adaptive learning, and thus learn faster and more efficiently. Typically, ICAI system includes the following smart: (1) can automatically generate a variety of problems and exercises; (2) be able to select the appropriate learning content and adjust instruction based on the progress of students with different levels of learning; (3) able to understand automatic basis for solving the problem of teaching content and generate an answer; generation and understanding (4) with a natural language in order to achieve more liberal teaching answering system, to improve the human-machine interaction initiative; (5) having a content of teaching explanation advisory capacity; (6) the student can diagnose errors, analyze why the error occurred and take appropriate corrective action; (7) can assess student learning behavior; (8) can continue to improve teaching strategies in teaching. ICAI is currently used in teaching have been many examples, such as building a network of expert mental health education system.

Intelligent Induct-learning System (TIS). Support services are important elements of modern computer network education system. Establish and maintain a highly efficient and flexible, strong support services subsystem is to effectively develop, manage and implement education programs to ensure that the computer network. Support services are designed to create a good learning environment, learners quickly and easily call a variety of resources on learning to accept a full-service, in order to be successful learning. Smart Agent is a distributed artificial intelligence in a large application, which aims to reduce the use of sophisticated software and people to perform the tedious task of burden, to maximize the efficiency of the current system based on intelligent Guidance technology to build intelligent AGENT [2] can vary guiding policy formulation and implementation, in line with the initiative to provide personalized service guidance that has some relevance guidance and forward-looking; science policy under the guidance of the guide automatically generate a variety of problems and exercises, rational planning, learning to adjust the content and progress, and have some diagnostic functions, diagnostics learning errors, analyze the causes and make improvements; automatically corrected by feedback guiding strategy to make it more in line with the learner's own knowledge base and recognition known characteristics.

Intelligent Simulation Technology (IST). In recent years, application of intelligent simulation technology developed CAI Courseware is expected to open up remote experimental teaching bottlenecks and easy to open the door of wisdom. IST intelligent simulation technology is evolving simulation environment and contribute to the development of a technology-driven, highly integrated artificial intelligence and simulation technology. IST strive to overcome the past, the traditional simulation models and modeling methods and modeling limitations arduous, monotonous interface issues and other aspects of the puzzling results, focusing on generalized simulation model, simulation model generation, intelligent simulation algorithm, intelligent simulation interface, the simulation results analysis, multi-database simulation software, integrated simulation language, simulation and other simulation information preprocessing techniques. IST input information preprocessing, simulation model generation, the flexibility of the effectiveness of simulation 
methods, simulation results analysis interpretation, etc., reflect the intelligence, adaptability and friendliness. Because smart alternative simulation system in a way to complete the simulation expert modeling, experimental design, understanding and evaluation of the simulation result of these steps require the expert to complete, and has some ability to learn. Therefore, the use of intelligent systems to develop experimental simulation courseware can greatly save manpower, reduce development costs and speed up development, shorten the development cycle.

Intelligent Hardware Network (IHN). Intelligent Network (Intelligent Network) referred IN, is a new research topic early 1980s the rise. "Smart" means here includes both the concept of "intelligence operations" and "intelligence service." Intelligent operation refers to the network system operation, maintenance and management of intelligence and intelligence services refers to the network system to provide users with some knowledge in the field of information processing or decision support to achieve some kind of demand. The computer network is the foundation of computer network hardware implementation of education, with the increasing popularity of human society and a high degree of information technology network, through the network of distance learning requires not only the integration of multimedia information processing capabilities, but also requires the network to deliver advanced information processing capabilities, That knowledge processing capabilities. On the current situation, the existing educational computer network gives it certain "intelligence" is a vision to enhance the choice is no lack of performance from the hardware itself.

Intelligent Network Examine System (INES). Currently paperless examination exam has become a major form of generalized paperless exam includes multiple links using computers to create and manage exam, test paper topic, examination and evaluation. It is not only from the form of the traditional pen and paper test of the revolution, and the connotation of examination and evaluation criteria also have a significant innovation. Many recent studies show that the use of AI techniques to improve the performance of paperless examination system has become a new research focus of which assigned to investigate the paper - making strategy more intelligent many scholars attention. Intelligent Test Paper not only low cost, high efficiency, confidentiality, continuous test paper consistency, and even in the more restrictions, the paper can still be generated automatically based on satisfying the principle of a given test paper.

\section{Conclusion}

The artificial intelligence technology in computer network teaching modern education will provide new development ideas, the overall improvement in network teaching environment, expand channels of learning services, improve the quality of teaching computer networks, and computer networks have the potential to revolutionize education in space-time break limits the overall strengthening of the teaching of the open network, network learning personalized, humane and intelligent, full implementation of student-centered teaching philosophy.

\section{References}

[1] Liu Guangzhong, Gao Jun. Computer Education, Vol. 1 (2014) No 53, p.25-26

[2] Zhao Ran, Wang Qunyong. Henan Science and Technology, Vol. 1 (2014) No 27, p.74-76

[3] Li Li. China Mining and Technology University, Vol. 30 (2004) No 19, p.144-145

[4] Wang Kuailiang. Mining machinery, Vol. 29 (2008) No 27, p.21-23

[5] Sun Jing. Computer Engineering and Applications, Vol. 8 (2003) No 27, p.83-84

(不加备注): 石家庄元氏县文化路与昌盛街交叉口东行 100 米路北梓墨文化 18630132561 张琼 